\title{
The Study of Cutting Conditions Effects on the Damping Process Using the Experimental Taguchi Method
}

\author{
Haikel Mejri ${ }^{1}$, Ziyed Sahraoui ${ }^{1}$ and Kamel Mehdi ${ }^{1,2}$ \\ ${ }^{1}$ URMSSDT, Engineering National High School of Tunis (ENSIT), University of Tunis (UT), 5 Avenue Taha Hussein P.B. 56 Bab Mnara \\ 1008 Tunis, Tunisia \\ ${ }^{2}$ Preparatory Institute for Engineering Studies El Manar (IPEIMa), University of Tunis EL Manar (UTM), PB. 24, 2092 Tunis, Tunisia
}

\begin{abstract}
This article focuses on determining the effects of cutting conditions and their interactions on the cutting process damping in the case of curvilinear milling. The tests were performed using a numerical model simulation that allows the prediction of cutting forces and damping. The effects and interactions are determined using the Taguchi experimental method. Analysis of variance (ANOVA) was performed to know the level of importance of the machining parameters on the cutting damping process. The results revealed that the Depth of cut $A_{p}$ "C" and cutting speed $V_{c}$ "B" have the most significant influence on the $\mathbf{C x x}$ and $\mathbf{C x y}$ process damping. The variations of tool diameter D "A" and clearance angle $\boldsymbol{\alpha}$ have remarkable effects on the process damping $\mathbf{C x x}$. The "BC" interaction has the greatest effect on the process damping $\mathbf{C x x}$ while the "AC" interaction has the greatest effect on the process damping Cxy.
\end{abstract}

\section{Introduction}

The comprehension of the various phenomena related to machining is necessary in order to propose models and methods which can predict the stability area. In most carried out researches, (which aims at the determination of the influence of the various cutting parameters), the researchers simplified or completely excluded the cutting process damping [1]. In fact, the experiments showed that for low cutting speeds, the cutting damping process is stable [2].

An important contribution on knowledge of the physical modeling of the cutting process was provided by Tlusty [3]. He showed with Ismail [4] that the cutting process damping affects the stability during the machining and it increases with the reduction of the cutting speed.

In 2012, Mehdi and Zghal [5] presented a numerical model allowing the prediction of cutting forces in peripheral milling process. They studied the effects of tool parameters (diameter, helix angle, number of teeth) on cutting process damping and cutting force distributions. In this model the cutting damping process was presented by the following matrix:

$$
\left[C_{c}\right]=\left[\begin{array}{ll}
C_{x x}(t) & C_{x y}(t) \\
C_{y x}(t) & C_{y y}(t)
\end{array}\right]
$$

Where:

$C_{x x}(t)=\sum_{j=1}^{N_{t}} \sum_{k=1}^{N_{d}} \delta_{k, j}\left[c_{t_{j}}(t)+c_{r_{j}}(t)\right] \cos \varphi_{j}(t) \sin \varphi_{j}(t)$

$$
\begin{aligned}
& \mathrm{C}_{\mathrm{xy}}(\mathrm{t})=\sum_{\mathrm{j}=1}^{\mathrm{N}_{\mathrm{t}}} \sum_{\mathrm{k}=1}^{\mathrm{N}_{\mathrm{d}}} \delta_{\mathrm{k}, \mathrm{j}}\left[\mathrm{c}_{\mathrm{t}_{\mathrm{j}}}(\mathrm{t}) \cos ^{2} \varphi_{\mathrm{j}}(\mathrm{t})-\mathrm{c}_{\mathrm{r}_{\mathrm{j}}}(\mathrm{t}) \sin ^{2} \varphi_{\mathrm{j}}(\mathrm{t})\right] \\
& \mathrm{C}_{\mathrm{yx}}(\mathrm{t})=\sum_{\mathrm{j}=1}^{\mathrm{N}_{\mathrm{t}}} \sum_{\mathrm{k}=1}^{\mathrm{N}_{\mathrm{d}}} \delta_{\mathrm{k}, \mathrm{j}}\left[\mathrm{c}_{\mathrm{r}_{\mathrm{j}}}(\mathrm{t}) \cos ^{2} \varphi_{\mathrm{j}}(\mathrm{t})-\mathrm{c}_{\mathrm{t}_{\mathrm{j}}}(\mathrm{t}) \sin ^{2} \varphi_{\mathrm{j}}(\mathrm{t})\right] \\
& C_{y y}(t)=-C_{x x}(t)
\end{aligned}
$$

Where:

$$
c_{r_{j}}(t) \text { and } c_{t_{j}}(t) \text { represent the cutting damping }
$$

factors in the thrust and tangential directions,

$\varphi_{j}(t)$ is the position angle of a point on the cutting edge of the $j^{\text {th }}$ helical flute,

$N_{t}$ is the number of the tool's teeth and $N_{d}$ is the number of the elementary cutting disks.

$\delta_{k, j}$ is equal to 1 , if the $\mathrm{j}^{\text {th }}$ tooth of the elementary $\mathrm{k}^{\text {th }}$ disk of the tool is in cut, and 0 if it's out of cut.

It is difficult to determine the cutting process damping in experiments, hence the importance of the numerical model simulation which was presented and validated experimentally. This model allowed us, initially, to determine the cutting process damping while using the experimental cutting values. Secondly, it permitted to study the variation of the damping process in function of cutting parameters thus enhancing the results already presented by researchers over the years by using the experimental plans method. In 2015, Li Xin and all [6] confirmed the importance of taking into account the 
cutting dumping process in their research on the machining of thin-walled workpiece.

The experimental plans method, is applicable to all kinds of engineering problems [7-10], and it brings a rigorous approach in the governance and the establishment of the tests, which enables us not to reason more by groping.

This paper comes to complete the previous work of Mehdi and Zghal [5]. It focuses on determining the effects of cutting conditions and their interactions on the cutting process damping in the case of curvilinear milling. The tests were performed using a numerical model simulation that allows the prediction of cutting forces and damping. The effects and interactions are determined using the Taguchi experimental method. Analysis of variance (ANOVA) was performed to know the level of importance of the machining parameters on the cutting damping process.

\section{Cutting process damping $\mathrm{Cxx}$}

In this part, we will use the parameters of cut for curvilinear machining allowed in table 1 and the material properties in table 2 .

Table 1. L16 standard table of settings applied to the simulation

\begin{tabular}{|c|c|c|c|c|}
\hline $\mathbf{N}^{\circ}$ test & $\begin{array}{c}\mathbf{D}(\mathbf{A}) \\
(\mathbf{m m})\end{array}$ & $\mathbf{V c}(\mathbf{B}) \mathbf{( m} / \mathbf{m i n})$ & $\begin{array}{c}\mathbf{A p}(\mathbf{C}) \\
(\mathbf{m m})\end{array}$ & $\begin{array}{c}\boldsymbol{\alpha}(\mathbf{D}) \\
(\mathbf{d e g r e e s})\end{array}$ \\
\hline 1 & 6 & 25 & 0,25 & 4 \\
\hline 2 & 6 & 25 & 0,25 & 15 \\
\hline 3 & 6 & 25 & 3 & 4 \\
\hline 4 & 6 & 25 & 3 & 15 \\
\hline 5 & 6 & 300 & 0,25 & 4 \\
\hline 6 & 6 & 300 & 0,25 & 15 \\
\hline 7 & 6 & 300 & 3 & 4 \\
\hline 8 & 6 & 300 & 3 & 15 \\
\hline 9 & 40 & 25 & 0,25 & 4 \\
\hline 10 & 40 & 25 & 0,25 & 15 \\
\hline 11 & 40 & 25 & 3 & 4 \\
\hline 12 & 40 & 25 & 3 & 15 \\
\hline 13 & 40 & 300 & 0,25 & 4 \\
\hline 14 & 40 & 300 & 0,25 & 15 \\
\hline 15 & 40 & 300 & 3 & 4 \\
\hline 16 & 40 & 300 & 3 & 15 \\
\hline
\end{tabular}

Figure 1 presents the results obtained by simulation of cutting forces. During each test, the measurement of cutting damping process Cxx was taken into account and we made the average.

Table 2. Material properties

\begin{tabular}{|l|c|}
\hline Steel & A60 \\
\hline $\operatorname{Rr}(\mathbf{M P a})$ & 570 \\
\hline $\operatorname{Re}(\mathbf{M P a})$ & 335 \\
\hline E $(\mathbf{M P a})$ & 200000 \\
\hline Density $\boldsymbol{\rho}(\mathbf{K g} / \mathbf{m 3})$ & 7850 \\
\hline Poisson's ratio & 0.29 \\
\hline
\end{tabular}

\subsection{Calculation of average effects of factors and the interactions effect}

The average value response of all factors of a $\mathrm{j}^{\text {th }}$ level is given equation (5).

$\bar{A}_{j}=\frac{1}{n} \sum_{i=1}^{n} R_{i j}$

Here, we have considered two levels $(j=1,2)$. Table 3 presents the average response to each factor level.

The overall average $\bar{T}$ of all the tests corresponds to the central point of average responses for the levels of each factor:

$\bar{T}=\frac{\bar{A} 1+\bar{A} 2}{2}=\frac{210.961+159}{2}=184.98 \mathrm{Ns} / \mathrm{m}$

The average effect of each factor level, appreciates relatively to the overall average. For example, the average effect of the factor A at the level 1 is calculated by the following equation:

$$
E_{A_{1}}=\bar{A} 1-\bar{T}=210.961-184.98=25.981 \mu \mathrm{m}
$$

Similarly, we calculate the average effect of the A at the level 2:

$E_{A_{2}}=\bar{A}_{2}-\bar{T}=159-184.98=-25.98 \mu m$

It is clear that: $\mathrm{EA}_{1}=-\mathrm{EA}_{2}$

Table 4 gives the average effects of all factors.

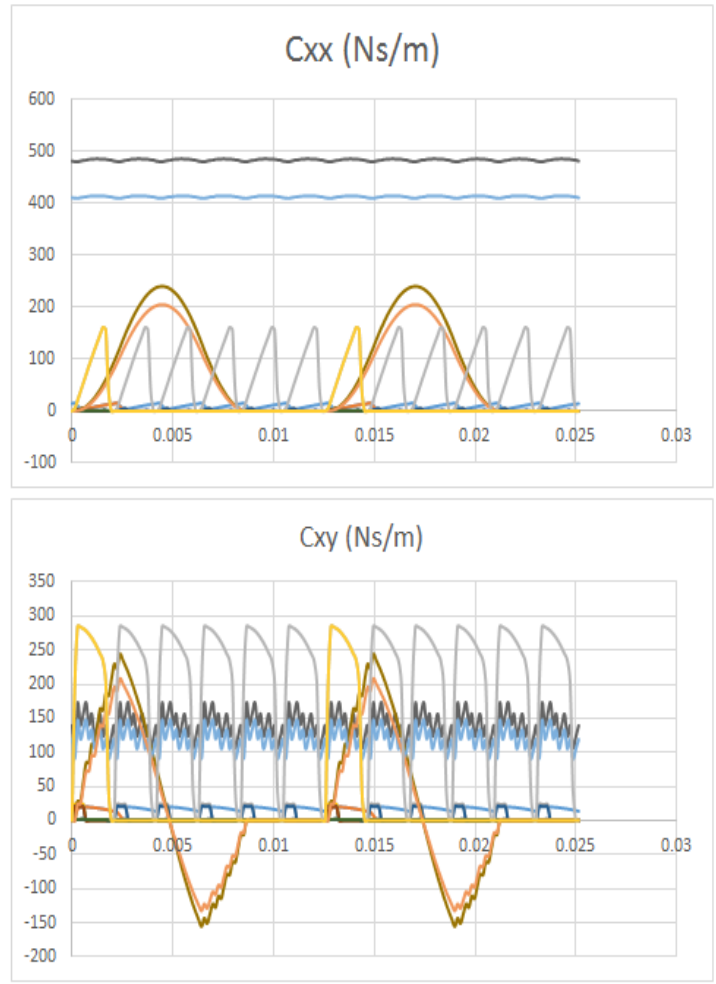

Figure 1. Effect of cutting parameters on cutting process damping (simulation) 
Table 3. Average values of factors responses for each level

\begin{tabular}{|c|c|c|}
\hline \multirow{2}{*}{ Fact } & \multicolumn{2}{|c|}{ Average response for each factor level } \\
\cline { 2 - 3 } & Lev. 1 & Lev. 2 \\
\hline A & 210.961 & 159.000 \\
\hline B & 266.469 & 103.493 \\
\hline C & 50.438 & 319.524 \\
\hline D & 151.689 & 218.273 \\
\hline
\end{tabular}

Table 4. Effect factors on the measured values.

\begin{tabular}{|c|c|c|}
\hline \multirow{2}{*}{ Fact } & \multicolumn{2}{|c|}{ Effect of factors on the measured values } \\
\cline { 2 - 3 } & Lev. 1 & Lev. 2 \\
\hline A & 25.981 & -25.981 \\
\hline B & 81.488 & -81.488 \\
\hline C & -134.543 & 134.543 \\
\hline D & -33.292 & 33.292 \\
\hline
\end{tabular}

\subsection{Calculation of the interactions}

By applying the general formula of calculation of the interactions, we find for $\mathrm{IA}_{1} \mathrm{~B}_{1}$ :

$I_{A_{1} B_{1}}=\bar{A}_{1} \bar{B}_{1}-E_{A_{1}}-E_{B 1}-\bar{T}$

The average response related to each of the combinations $\mathrm{AB}$ is calculated using the same principle as for the following factors:

$\bar{A}_{1} \bar{B}_{1}=\frac{160.86+160.86+204.76+414.11}{4}=235.148 \mathrm{Ns} / \mathrm{m}$

Table 5 gives the average values of the interactions

Table 5. Average values of interactions.

\begin{tabular}{|c|c|c|c|}
\hline \multicolumn{4}{|c|}{ Average values of interactions } \\
\hline $\mathbf{A}_{\mathbf{1}} \mathbf{B}_{\mathbf{1}}$ & $\mathbf{A}_{\mathbf{1}} \mathbf{B}_{\mathbf{2}}$ & $\mathbf{A}_{\mathbf{2}} \mathbf{B}_{\mathbf{1}}$ & $\mathbf{A}_{\mathbf{2}} \mathbf{B}_{\mathbf{2}}$ \\
\hline 235.148 & 186.775 & 297.790 & 20.210 \\
\hline $\mathbf{A}_{\mathbf{1}} \mathbf{C}_{\mathbf{1}}$ & $\mathbf{A}_{\mathbf{1}} \mathbf{C}_{\mathbf{2}}$ & $\mathbf{A}_{\mathbf{2}} \mathbf{C}_{\mathbf{1}}$ & $\mathbf{A}_{\mathbf{2}} \mathbf{C}_{\mathbf{2}}$ \\
\hline 85.905 & 336.018 & 14.970 & 303.030 \\
\hline $\mathbf{A}_{\mathbf{1}} \mathbf{D}_{\mathbf{1}}$ & $\mathbf{A}_{\mathbf{1}} \mathbf{D}_{\mathbf{2}}$ & $\mathbf{A}_{\mathbf{2}} \mathbf{D}_{\mathbf{1}}$ & $\mathbf{A}_{\mathbf{2}} \mathbf{D}_{\mathbf{2}}$ \\
\hline 153.166 & 283.071 & 149.250 & 168.750 \\
\hline $\mathbf{B}_{\mathbf{1}} \mathbf{C}_{\mathbf{1}}$ & $\mathbf{B}_{\mathbf{1}} \mathbf{C}_{\mathbf{2}}$ & $\mathbf{B}_{\mathbf{2}} \mathbf{C}_{\mathbf{1}}$ & $\mathbf{B}_{\mathbf{2}} \mathbf{C}_{\mathbf{2}}$ \\
\hline 73.092 & 493.309 & 51.642 & 187.243 \\
\hline $\mathbf{B}_{\mathbf{1}} \mathbf{D}_{\mathbf{1}}$ & $\mathbf{B}_{\mathbf{1}} \mathbf{D}_{\mathbf{2}}$ & $\mathbf{B}_{\mathbf{2}} \mathbf{D}_{\mathbf{1}}$ & $\mathbf{B}_{\mathbf{2}} \mathbf{D}_{\mathbf{2}}$ \\
\hline 230.550 & 302.388 & 72.828 & 134.158 \\
\hline $\mathbf{C}_{\mathbf{1}} \mathbf{D}_{\mathbf{1}}$ & $\mathbf{C}_{\mathbf{1}} \mathbf{D}_{\mathbf{2}}$ & $\mathbf{C}_{\mathbf{2}} \mathbf{D}_{\mathbf{1}}$ & $\mathbf{C}_{\mathbf{2}} \mathbf{D}_{\mathbf{2}}$ \\
\hline 50.438 & 34.663 & 252.940 & 386.108 \\
\hline
\end{tabular}

Then, from this table, we get:

$I_{A_{1} B_{1}}=235.148-25.98-81.488-184.98=-57.30 \mu m(11)$

And in the same way we calculate the other interactions. The values of these interactions are given in table 6 .
Table 6. Effect of interactions on the measured values

\begin{tabular}{|c|c|c|c|}
\hline \multicolumn{4}{|c|}{ Effect of interactions on the measured values } \\
\hline $\mathbf{A}_{\mathbf{1}} \mathbf{B}_{\mathbf{1}}$ & $\mathbf{A}_{\mathbf{1}} \mathbf{B}_{\mathbf{2}}$ & $\mathbf{A}_{\mathbf{2}} \mathbf{B}_{\mathbf{1}}$ & $\mathbf{A}_{\mathbf{2}} \mathbf{B}_{\mathbf{2}}$ \\
\hline-57.302 & 57.302 & 57.302 & -57.302 \\
\hline $\mathbf{A}_{\mathbf{1}} \mathbf{C}_{\mathbf{1}}$ & $\mathbf{A}_{\mathbf{1}} \mathbf{C}_{\mathbf{2}}$ & $\mathbf{A}_{\mathbf{2}} \mathbf{C}_{\mathbf{1}}$ & $\mathbf{A}_{\mathbf{2}} \mathbf{C}_{\mathbf{2}}$ \\
\hline 9.487 & -9.487 & -9.487 & 9.487 \\
\hline $\mathbf{A}_{\mathbf{1}} \mathbf{D}_{\mathbf{1}}$ & $\mathbf{A}_{\mathbf{1}} \mathbf{D}_{\mathbf{2}}$ & $\mathbf{A}_{\mathbf{2}} \mathbf{D}_{\mathbf{1}}$ & $\mathbf{A}_{\mathbf{2}} \mathbf{D}_{\mathbf{2}}$ \\
\hline-24.504 & -0.005 & 23.542 & -23.542 \\
\hline $\mathbf{B}_{\mathbf{1}} \mathbf{C}_{\mathbf{1}}$ & $\mathbf{B}_{\mathbf{1}} \mathbf{C}_{\mathbf{2}}$ & $\mathbf{B}_{\mathbf{2}} \mathbf{C}_{\mathbf{1}}$ & $\mathbf{B}_{\mathbf{2}} \mathbf{C}_{\mathbf{2}}$ \\
\hline-58.834 & 92.297 & 82.692 & -50.793 \\
\hline $\mathbf{B}_{\mathbf{1}} \mathbf{D}_{\mathbf{1}}$ & $\mathbf{B}_{\mathbf{1}} \mathbf{D}_{\mathbf{2}}$ & $\mathbf{B}_{\mathbf{2}} \mathbf{D}_{\mathbf{1}}$ & $\mathbf{B}_{\mathbf{2}} \mathbf{D}_{\mathbf{2}}$ \\
\hline-2.627 & 2.627 & 2.627 & -2.627 \\
\hline $\mathbf{C}_{\mathbf{1}} \mathbf{D}_{\mathbf{1}}$ & $\mathbf{C}_{\mathbf{1}} \mathbf{D}_{\mathbf{2}}$ & $\mathbf{C}_{\mathbf{2}} \mathbf{D}_{\mathbf{1}}$ & $\mathbf{C}_{\mathbf{2}} \mathbf{D}_{\mathbf{2}}$ \\
\hline 33.292 & -0.025 & -33.292 & 33.292 \\
\hline
\end{tabular}

\subsection{Discussion}

The curves illustrated in figures 2 and 3 represent all the effects and interactions on the cutting damping process Cxx. From these figures, we can see that:

-The pass depth (C) and the cutting speed (B) have the most significant influence on the process damping $\mathrm{Cxx}$. Indeed, if we increase the cutting speed, the process damping Cxx decrease.

-By against, if we increase the depth of cutting, the process damping $\mathrm{Cxx}$ increase.

-The variation of the tool diameter (D) and the variation of the clearance angle $(\alpha)$ have an effect on cutting damping process Cxx. However, the effect of both factors remains lowers than that the depth of cutting and cutting speed.

-The $\mathrm{BC}$ interaction has the greatest effect on the cutting damping process $\mathrm{Cxx}, \mathrm{AB}$ interaction also exhibits a significant effect. This is a reason for which it must be taken into consideration.

-The CD interaction is intense, although its effect is lower than that of $\mathrm{BC}$ and $\mathrm{AB}$.

-Interactions $\mathrm{AB}, \mathrm{AC}, \mathrm{AD}$ and $\mathrm{BC}$ are low.

-The BD interaction is null, then it has no effect on the cutting damping process $\mathrm{Cxx}$.

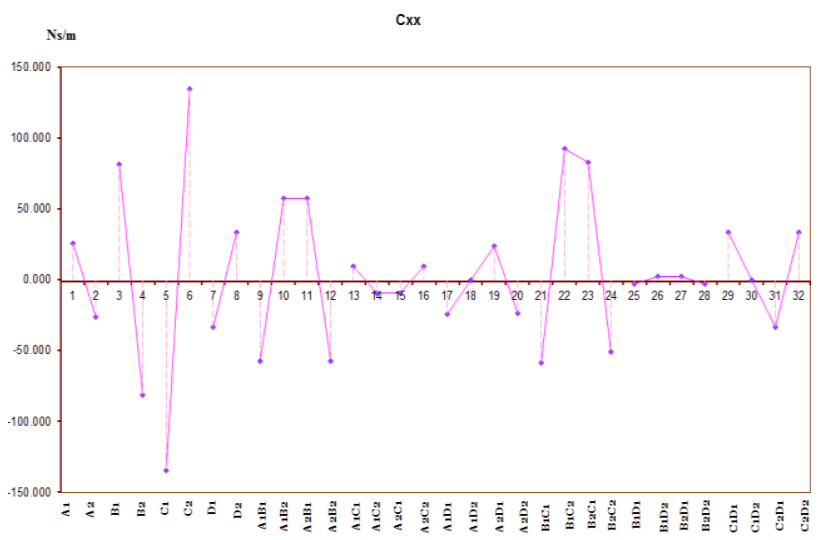

Figure 2. Effect of factors and interactions on Cxx 


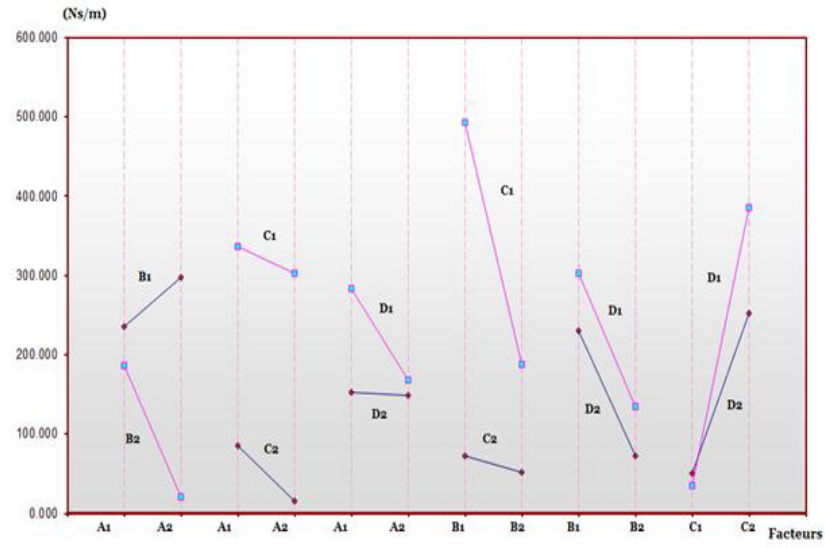

Figure 3. Effect of interactions on Cxx cutting damping

\section{Cutting process damping $\mathbf{C x y}$}

Like in the case of cutting process damping Cxx, the same approach has been adopted to determine the tables of the average response, the effect of factors on the measured values, the average values of the interactions and the effect of the interactions on the measured values (tables 7 , 8,9 and 10).

Table 7. Average values of factors responses for each level.

\begin{tabular}{|c|c|c|}
\hline \multirow{2}{*}{ Fact } & \multicolumn{2}{|c|}{ Average values of factors for each level } \\
\cline { 2 - 3 } & Lev. 1 & Lev. 2 \\
\hline A & 168.333 & 140.233 \\
\hline B & 244.599 & 63.967 \\
\hline C & 82.679 & 225.886 \\
\hline D & 152.224 & 156.342 \\
\hline
\end{tabular}

Table 8. Effect of factors on the measured values.

\begin{tabular}{|c|c|c|}
\hline \multirow{2}{*}{ Fact } & \multicolumn{2}{|c|}{ Effect of factors on the measured values } \\
\cline { 2 - 3 } & Lev, 1 & Lev, 2 \\
\hline A & 14.050 & -14.050 \\
\hline B & 90.316 & -90.316 \\
\hline C & -71.604 & 71.604 \\
\hline D & -2.059 & 2.059 \\
\hline
\end{tabular}

Table 9. Average values of interactions

\begin{tabular}{|c|c|c|c|}
\hline \multicolumn{4}{|c|}{ Average values of interactions } \\
\hline $\mathbf{A}_{\mathbf{1}} \mathbf{B}_{\mathbf{1}}$ & $\mathbf{A}_{\mathbf{1}} \mathbf{B}_{\mathbf{2}}$ & $\mathbf{A}_{\mathbf{2}} \mathbf{B}_{\mathbf{1}}$ & $\mathbf{A}_{\mathbf{2}} \mathbf{B}_{\mathbf{2}}$ \\
\hline 231.560 & 105.106 & 257.638 & 22.828 \\
\hline $\mathbf{A}_{\mathbf{1}} \mathbf{C}_{\mathbf{1}}$ & $\mathbf{A}_{\mathbf{1}} \mathbf{C}_{\mathbf{2}}$ & $\mathbf{A}_{\mathbf{2}} \mathbf{C}_{\mathbf{1}}$ & $\mathbf{A}_{\mathbf{2}} \mathbf{C}_{\mathbf{2}}$ \\
\hline 143.341 & 193.325 & 22.018 & 258.448 \\
\hline $\mathbf{A 1 D} \mathbf{1}$ & $\mathbf{A}_{\mathbf{1}} \mathbf{D}_{\mathbf{2}}$ & $\mathbf{A}_{\mathbf{2}} \mathbf{D}_{\mathbf{1}}$ & $\mathbf{A}_{\mathbf{2}} \mathbf{D}_{\mathbf{2}}$ \\
\hline 184.883 & 151.783 & 119.565 & 160.900 \\
\hline $\mathbf{B 1 C} \mathbf{1}$ & $\mathbf{B}_{\mathbf{1}} \mathbf{C}_{\mathbf{2}}$ & $\mathbf{B}_{\mathbf{2}} \mathbf{C}_{\mathbf{1}}$ & $\mathbf{B}_{\mathbf{2}} \mathbf{C}_{\mathbf{2}}$ \\
\hline 152.638 & 336.560 & 12.721 & 115.213 \\
\hline $\mathbf{B} 1 \mathbf{D} \mathbf{1}$ & $\mathbf{B}_{\mathbf{1}} \mathbf{D}_{\mathbf{2}}$ & $\mathbf{B}_{\mathbf{2}} \mathbf{D}_{\mathbf{1}}$ & $\mathbf{B}_{\mathbf{2}} \mathbf{D}_{\mathbf{2}}$ \\
\hline 231.945 & 257.253 & 72.503 & 55.431 \\
\hline $\mathbf{C}_{\mathbf{1}} \mathbf{D}_{\mathbf{1}}$ & $\mathbf{C}_{\mathbf{1}} \mathbf{D}_{\mathbf{2}}$ & $\mathbf{C}_{\mathbf{2}} \mathbf{D}_{\mathbf{1}}$ & $\mathbf{C}_{\mathbf{2}} \mathbf{D}_{\mathbf{2}}$ \\
\hline 82.678 & 82.681 & 221.770 & 230.003 \\
\hline
\end{tabular}

Table 10. Effect of the interactions on the measured values

\begin{tabular}{|c|c|c|c|}
\hline \multicolumn{4}{|c|}{ Effect of the interactions on the measured value } \\
\hline $\mathbf{A}_{\mathbf{1}} \mathbf{B}_{\mathbf{1}}$ & $\mathbf{A}_{\mathbf{1}} \mathbf{B}_{\mathbf{2}}$ & $\mathbf{A}_{\mathbf{2}} \mathbf{B}_{\mathbf{1}}$ & $\mathbf{A}_{\mathbf{2}} \mathbf{B}_{\mathbf{2}}$ \\
\hline-27.089 & 27.089 & 27.089 & -27.089 \\
\hline $\mathbf{A}_{\mathbf{1}} \mathbf{C}_{\mathbf{1}}$ & $\mathbf{A}_{\mathbf{1}} \mathbf{C}_{\mathbf{2}}$ & $\mathbf{A}_{\mathbf{2}} \mathbf{C}_{\mathbf{1}}$ & $\mathbf{A}_{\mathbf{2}} \mathbf{C}_{\mathbf{2}}$ \\
\hline 46.612 & -46.612 & -46.612 & 46.612 \\
\hline $\mathbf{A 1 D} \mathbf{1}$ & $\mathbf{A}_{\mathbf{1}} \mathbf{D}_{\mathbf{2}}$ & $\mathbf{A}_{\mathbf{2}} \mathbf{D}_{\mathbf{1}}$ & $\mathbf{A}_{\mathbf{2}} \mathbf{D}_{\mathbf{2}}$ \\
\hline 18.609 & -18.609 & -18.609 & 18.609 \\
\hline $\mathbf{B 1 C} \mathbf{1}$ & $\mathbf{B}_{\mathbf{1}} \mathbf{C}_{\mathbf{2}}$ & $\mathbf{B}_{\mathbf{2}} \mathbf{C}_{\mathbf{1}}$ & $\mathbf{B}_{\mathbf{2}} \mathbf{C}_{\mathbf{2}}$ \\
\hline-20.358 & 20.358 & 20.358 & -20.358 \\
\hline $\mathbf{B} \mathbf{1 D} \mathbf{1}$ & $\mathbf{B}_{\mathbf{1}} \mathbf{D}_{\mathbf{2}}$ & $\mathbf{B}_{\mathbf{2}} \mathbf{D}_{\mathbf{1}}$ & $\mathbf{B}_{\mathbf{2}} \mathbf{D}_{\mathbf{2}}$ \\
\hline-10.595 & 10.595 & 10.595 & -10.595 \\
\hline $\mathbf{C}_{\mathbf{1}} \mathbf{D}_{\mathbf{1}}$ & $\mathbf{C}_{\mathbf{1}} \mathbf{D}_{\mathbf{2}}$ & $\mathbf{C}_{\mathbf{2}} \mathbf{D}_{\mathbf{1}}$ & $\mathbf{C}_{\mathbf{2}} \mathbf{D}_{\mathbf{2}}$ \\
\hline 2.058 & -2.057 & -2.058 & 2.058 \\
\hline
\end{tabular}

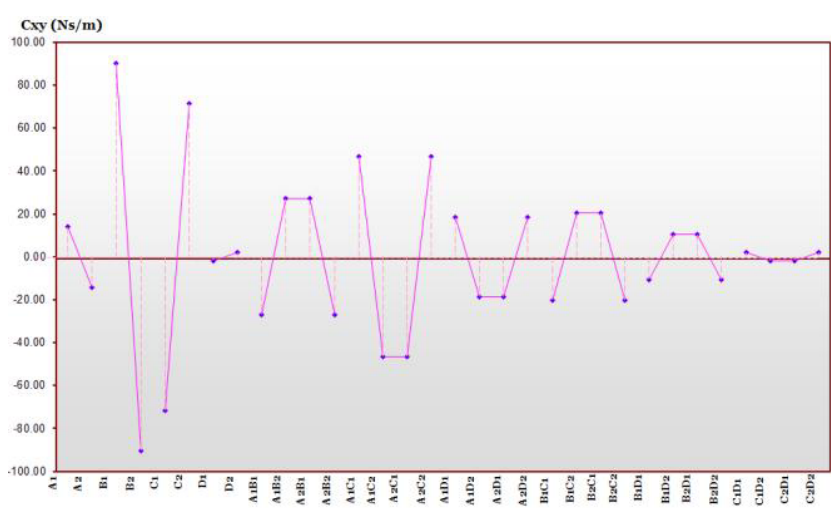

Figure 4. Effect of factors and interactions on Cxy

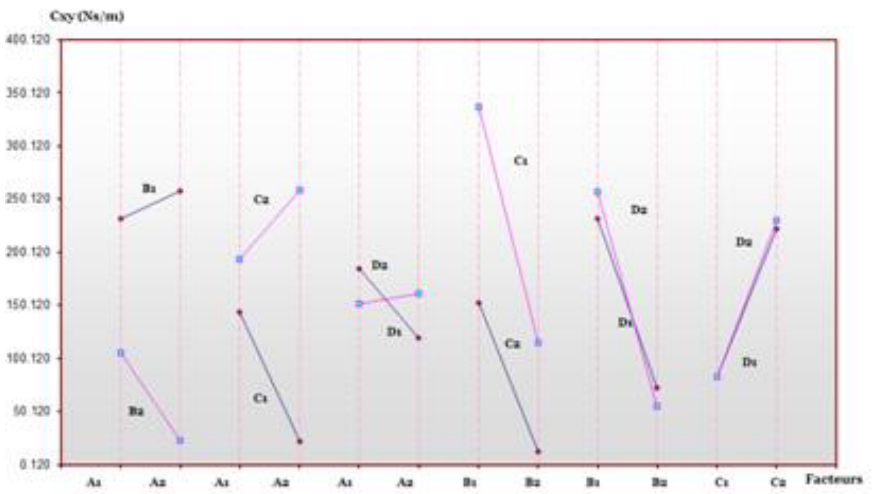

Figure 5. Effect of interactions on Cxy damping

The curves illustrated in figures 4 and 5 represent all the effects and interactions on the Cxy cutting damping process. From these figures we can see that:

-The cutting depth (C) and the cutting speed (B) have the most significant influence on the cutting damping process Cxy. In fact if we increase the cutting speed, the cutting damping process Cxy decrease. On the other hand, if we increase the depth of cut, the cutting damping process Cxy increase. It is noticed that the effect of the cutting speed (B) is more important.

-The cutting damping process Cxy passes by negative values by increasing the cutting depth. 
-The interaction $\mathrm{AC}$ has the greatest effect on the cutting damping process $\mathrm{Cxy}$, interactions $\mathrm{AB}, \mathrm{AD}$ and $\mathrm{BC}$ also present a significant effect.

-The interactions $\mathrm{AD}, \mathrm{BD}$ and $\mathrm{CD}$ are intense; despite their effects remain lower than that of $\mathrm{AC}$.

-Interactions $\mathrm{AB}, \mathrm{AC}$ and $\mathrm{BC}$ are not very significant.

\section{Conclusions}

In this paper, a Taguchi experimental plan was used. This method allows the limitation of the number of necessary and sufficient tests to study the cutting damping process in curvilinear milling was used.

We have succeeded in identify the main influential machining parameters on the cutting damping process. Such as the tool diameter (D), the cutting depth (ap), the cutting speed $(\mathrm{Vc})$ and clearance angle $(\alpha)$.

The simulation results have allowed us to conclude that:

-The cutting depth (C) and the cutting speed (B) have the most significant influence on the cutting damping process Cxx and Cxy.

-The variation of the tool diameter (D) and the variation of the clearance angle $(\alpha)$ act in a remarkable manner on the cutting damping process $\mathrm{Cxx}$, but they have a less significant effect on the cutting damping process Cxy.

-The BC interaction has the greatest effect on the cutting damping process $\mathrm{Cxx}$, the $\mathrm{AB}$ interaction of same has a significant effect.

-AC interaction has the greatest effect on the cutting damping process $\mathrm{Cxy}$, interactions $\mathrm{AB}, \mathrm{AD}$ and $\mathrm{BC}$ of same have a significant effect. This is one reason why we must take into consideration.

Finally, it is important to insist on the major role of numerical model simulation, with it we have predicted the cutting damping process in curvilinear milling. The future work of this research is the study of the effect of cutting parameters and tool parameters and their interactions on the cutting forces.

\section{References}

1. C.Y. Huang, J.J. Wang, "Mechanistic Modeling of Process Damping in Peripheral Milling", Transactions of the ASME, Journal of Manufacturing Science and Engineering, Vol. 129, pp. 12-20 (2007).

2. D.Y. Jang, A. Seireg, "Tool Natural Frequency as the Control Parameter for Surface Roughness" Machine Vibration, Vol. 1, pp. 147-154). (1992)

3. J. Tlusty, "Dynamics of high speed milling" Transactions of the ASME, Journal of Engineering for Industry, Vol. 108, pp. 59-67 (1986).

4. J. Tlusty, F. Ismail, "Special Aspects of Chatter in Milling" Transactions of the ASME, Journal of Engineering for Industry, Vol. 105, pp. 24-32 (1983).

5. K. Mehdi, A. Zghal, "Modelling Cutting Force Including Thrust and Tangential Damping in Peripheral Milling Process" Int. J. Machining and Machinability of Materials, Vol. 12, No. 3, (2012)

6. Xin Li, Wei Zhao, Liang Li, Ning He, and ShengWei Chi"Modeling and Application of Process Damping in Milling of Thin-Walled Workpiece Made of Titanium Alloy" Hindawi Publishing Corporation Shock and Vibration Vol. 2015, Article ID 431476, 12 pages, http://dx.doi.org/10.1155/2015/431476.

7. J. GOUPY. "Plans d'expériences" Techniques de l'ingénieur. Traité Analyse Chimique et Caractérisation, P 230, p. 1-20. (1992).

8. J.GOUPY. "Introduction aux plans d'expériences" Dunod. Paris. Pages 303. (2001).

9. J.GOUPY. "Pratiquer les Plans d'Expériences". Dunod. Paris. 560 pages. (2005).

10. J. GOUPY, L. CREIGHTON, "Introduction aux plans d'expériences", DUNOD, (2006). 